Journal of Engineering and Applied Sciences 14 (14): 4754-4760, 2019

ISSN: 1816-949X

(C) Medwell Journals, 2019

\title{
Face Detection Methods: A Comparative Study Between Viola-Jones and Skin Color Detection
}

\author{
Maryam Mahmood Hussein and Ammar Hussein Mutlag \\ College of Electrical Engineering Technical, Middle Technical University, Baghdad, Iraq
}

\begin{abstract}
Face identification is an important factor in the field of Personal Computer (PC) vision, since, it is the initial phase in different applications like face acknowledgment, military knowledge and reconnaissance, human PC association and so on. In this study, the execution examination has been made of 2 different algorithms for face identification in color images. The 1st algorithm is the CIELAB color model. In this algorithm, the knowledge of geometrical properties of human face has been utilized to determine if the skin area is human face or not. The 2nd algorithm is Viola-Jones algorithm. This method is used to identify face from entire image. The main concepts engaged with Viola-Jones are Harr features integral image, AdaBoost learning and cascade classifier. The overall comparison for both algorithms have applied under MATLAB environment. For fair comparison, 66 images are applied for both algorithms. The success of the Viola-Jones algorithm is $87.87 \%$ meanwhile the success of the CIELAB algorithm is $46.96 \%$. The outcome exhibits that the Viola-Jones method gives better outcomes contrasted the other method.
\end{abstract}

Key words: Face detection, Viola-Jones, skin color detection, CIELAB color model, MATLAB, geometrical, comparison

\section{INTRODUCTION}

At present, the surveillance requirement is specifically related to security perspectives in everyday life. Beginning from the field of managing an account, workplaces and some open offices, for example, airplane, terminals stations and not rarely a few houses start to apply this type of observing. This type of observing is constantly founded on the requirement for consistent checking and recording of all activities occurring in an area with the trust that when something basic occurs it tends to be instantly recognized and addressed, for example, the likelihood of criminals or different items that may meddle with the security of a building. Besides, to screen each development of this object it takes a face recognition framework that can recognize each face of human that goes into a working under checking. Face detection is the initial step required for facial acknowledgment frameworks (Raya et al., 2017). It is the issue of deciding if a sub-window of a picture contains a face (Raghuvanshi and Agrawal, 2012), also deciding their areas. It is viewed as a testing errand as a result of numerous varieties in the images and in addition the countenances inside them. Numerous components influence the way toward distinguishing appearances for example, scale area, poses, expression and lighting conditions (Dasan et al., 2015). A few strategies and calculations have been utilized for face detecting incorporate skin color detection, Harr classifier, neural network techniques, Viola-Jones algorithm and AdaBoost training (Raya et al., 2017; Anchit and Mathur, 2014; Luh, 2014). Skin color is great element for identification the human face. It has turned out to be helpful and strong prompt for face detection, limitation and following. It is helpful in human PC communication, human face acknowledgment framework and vision based motion acknowledgment. Skin color division ends up solid, if chrominance segment utilized for division. The technique is picking which dispense with the variety in luminance part. Detection of skin color faces numerous issues like distinctive cameras produces diverse shading esteems not with standing for same individual under same light molding. The luminance part has more variety which is most vital issue among current skin identification framework that genuinely debases the execution. The color model chosen for skin color division decide how effectively skin color model strategy distinguish the skin color. A hindrance of color prompt is its affectability to brightening color change particularly in Red-Green-Blue (RGB) color model. One approach to conquer this issue is change the image implies change RGB image into other color model whose luminance and chrominance are

Corresponding Author: Maryam Mahmood Hussein, College of Electrical Engineering Technical, Middle Technical University, Baghdad, Iraq 
isolated (Kaur and Kranthi, 2012). Generally, 3 basic stages pass through the face detection process using the color of the skin which are speaking to the image pixels in a reasonable color model, demonstrating the skin and non-skin pixels utilizing a proper distribution and arranging the demonstrated distributions (Luh, 2014). The skin detection considered the oldest method of face detection (Raghuvanshi and Agrawal, 2012) and wide assortment of studies have been proposed on it (Luh, 2014). However, the most well-known and powerful method for face location is Viola-Jones (Dasan et al., 2015). The Viola-Jones object location system is the first object discovery structure to give competitive object discovery rates continuously proposed in 2001 by Paul Viola and Michael Jones. This face identification structure is fit for preparing images to a great degree quickly while accomplishing high identification rates (Gupta and Sharma, 2014). This study attempt to compare between Viola-Jones and skin color based face detection. The comparison has been achieved under MATLAB environment to validate the performance of both algorithms.

\section{MATERIALS AND METHODS}

Viola-Jones algorithm: Many face detection algorithms have been mentioned in the literature. One of the most important method is the Viola-Jones algorithm (Deshpande and Ravishankar, 2017). Paul Viola and Michael Jones had proposed this algorithm in year 2001. It was gone for focusing on the issue of face discovery, however can likewise be prepared for recognizing diverse object detection (Tikoo and Malik, 2017). Viola-Jones object identifier becomes well known because of its open source usage in the open computer vision library (Gupta and Tiwari, 2014). It has the benefit of high location rate and great continuous execution ( $\mathrm{Lv}$ et al., 2017). It contains 4 phases to be specific. These phases will describe in the following sections.

Harr features: The extraction of features from images is the significant part in this procedure which utilizes Haar classifier. Haar features can determines the existence of any feature in the given picture. Every feature restores a single esteem which is given by subtracting of aggregate of the pixels in the white district from the entirety of pixels in the dark locale as described in Eq. 1:

$\mathrm{F}(\mathrm{x})=\sum$ (pixels in back area)-

$\sum$ (pixels in white area)

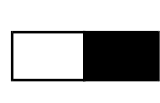

Type 1

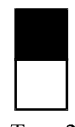

Type 2

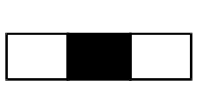

Type 3

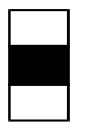

Type 4

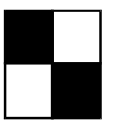

Type 5
Fig. 1: A few of the Haar features

\begin{tabular}{|l|l|l|l|}
\hline 1 & 4 & 3 & 8 \\
\hline 2 & 6 & 4 & 2 \\
\hline 1 & 9 & 5 & 6 \\
\hline 7 & 5 & 1 & 9 \\
\hline
\end{tabular}

Fig. 2: Original image

\begin{tabular}{|c|c|c|c|}
\hline 1 & 5 & 8 & 16 \\
\hline 3 & 13 & 20 & 30 \\
\hline 4 & 23 & 39 & 51 \\
\hline 11 & 35 & 48 & 73 \\
\hline
\end{tabular}

Fig. 3: Integral image

A few of the Haar features that can be utilized in the recognition of faces are given in Fig. 1 (Nehru and Padmavathi, 2017).

Integral image: For quickly feature extraction, another image portrayal called integral image has been utilized (Viola and Jones, 2001). For example, the original image is shown in Fig. 2, therefore, the value at any location (x,y) of integral image is the sum of pixels values above and left of this location in original image as can be shown in Fig. 3 (Gupta and Tiwari, 2014).

After the outcomes acquired from the computation of integral image at that point will be ascertained for a specific area. To ascertain the quantity of pixels in area D shown in Fig. 4, Eq. 2 is utilized. The values of L1-L 4 are $13,30,35$ and 73 , respectively. Therefore, the value of the area $\mathrm{D}$ according to $\mathrm{Eq} .2$ is 21 pixels (Raya et al., 2017). 


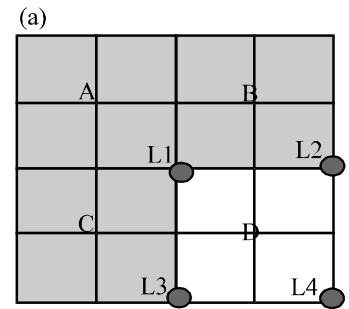

(b)
\begin{tabular}{|c|c|c|c|}
\hline 1 & 5 & 8 & 16 \\
\hline 3 & 13 & 20 & 30 \\
\hline 4 & 23 & 39 & 51 \\
\hline 11 & 35 & 48 & 73 \\
\hline
\end{tabular}

Fig. 4: Summation of pixels in specific region

$$
\mathrm{D}=(\mathrm{L} 1+\mathrm{L} 4)-(\mathrm{L} 2+\mathrm{L} 3)
$$

AdaBoost algorithm: Voila-Jones calculation utilizes a $24 \times 24$ window as the base window size to begin assessing Harr features in some random picture (Gupta and Tiwari, 2014). By using this window, around 160000 features can be computed which is incomprehensible basically (Nehru and Padmavathi, 2017). This is why AdaBoost is used. AdaBoost algorithm is a machine learning calculation which encourages us to locate the best classifiers among the 160000 features. These classifiers are the frail classifiers. AdaBoost creates a solid classifier as a direct mix of these weighted frail classifiers as appeared as (Gupta and Tiwari, 2014):

$$
F(x)=\operatorname{alf} 1(x)+a 2 f 2(x)+a 3 f 3(x)+\ldots,
$$

where, $F(x)$ represents the solid classifier while $\mathrm{fl}(\mathrm{x}), \mathrm{f} 2(\mathrm{x})$ and so on represents frail classifiers, a1, a2 and so on are weighted quantities and $\mathrm{x}$ is an image (Mehta and Tomar, 2016).

Cascade classifier: Cascade classifier is a strategy for consolidating progressively strong classifiers in cascade stages which drastically expands the speed of the locator by concentrating consideration on promising districts of the image (Viola and Jones, 2001). Non-face sub window can be rapidly neglecting using cascading. The cascade classifier neglects the sub window if it not passed the first stage. On the off chance that it go than send it to next stage which is more confused than past one. In the event that sub window passed all the stages, this implies a face is recognized as shown on Fig. 5 (Dabhi and Pancholi, 2016).

Color model for skin color location: A skin color is displaying the motivation to utilize skin color examination. For beginning grouping of a picture into likely face and no face areas comes from various straight forward yet great attributes of skin color. Firstly, skin color is less complex

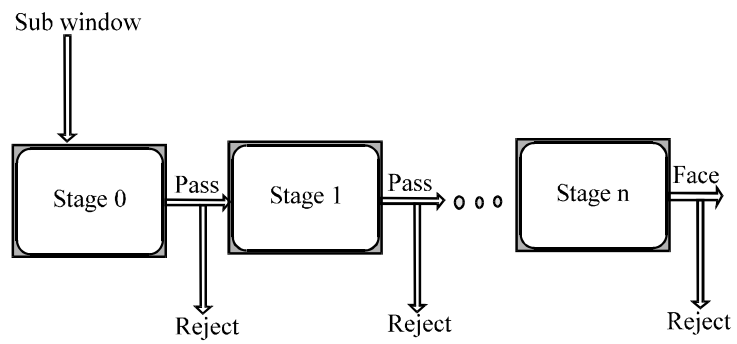

Fig. 5: Cascade of stages

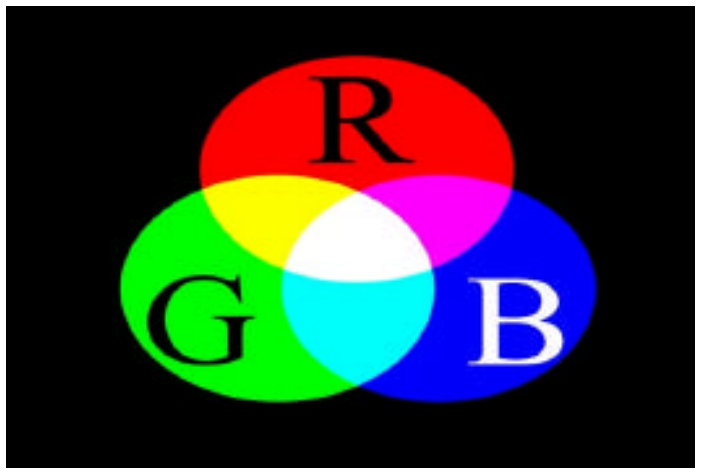

Fig. 6: RGB color model

than handling some other facial components. Secondly, under certain lighting conditions it is introduction invariant. The significant distinction between skin tones is force because of changing lighting conditions and distinctive human race. One essential factor that ought to be considered while building a measurable model for color is the choice of a color model (Prashanth and Shashidhara, 2014). Color model is a numerical representation of color data as 3 or 4 distinctive color parts (Shaik et al., 2015). Diverse color models that have been utilized for the color division is finished by utilizing chrominance segment, since, it is normal that skin color division may turn out to be more solid to lighting varieties if luminance segment is disposed of (Kaur and Kranthi, 2012). Coming up is a depiction of the 2 well-known color models which are RGB and CIELAB.

RGB color model: RGB color model comprises of the 3 added substance primaries red, green and blue. These otherworldly parts are utilized to create any other color (Prashanth and Shashidhara, 2014). It is a standout amongst the most broadly utilized color for handling and processing of digital image information. Nonetheless, high relationship between channels, blending of chrominance and luminance information make RGB not an extremely ideal decision for color investigation and color based detection methods (Manjare and Chougule, 2013) (Fig. 6). 


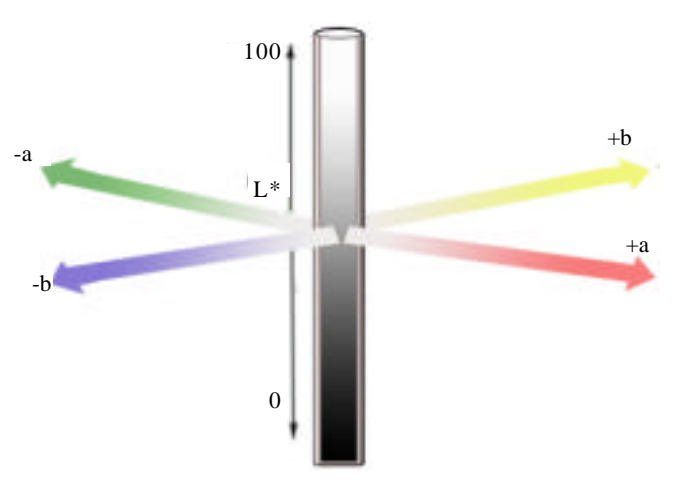

Fig. 7: CIELAB color model

CIELAB color model: LAB color is a sort of color space which is distributed in 1976 by International Commission on Illumination (CIE). It is a numerical color space dependent on light sensitivity of peoples visual spectrum. It is incorporates all visible spectrum in hypothesis, so, color data in different spaces including RGB space can be portray in lab space (Fan and Wang, 2013). The CIELAB color model consists of triple components which are L, A and $\mathrm{B}$ as can be shown in Fig. 7 the $(\mathrm{L})$ is determined the lighting information meanwhile the $(\mathrm{AB})$ are determined the chroma information.

The CIELAB color model has been utilized in this study where it is more robust than the RGB Model (Kaur and Kranthi, 2012). The CIELAB color model will be explained in the next study.

Skin color location using CIELAB color model: This part clarifies the method utilized for the skin identification in color pictures utilizing CIELAB color model.

Skin color segmentation: The process of skin color division expels all non-skin pixels and keeping the skin-pixels, since, an essential strategy for recognizing faces is usually feature of skin color. The color dispersion is gathered in the little district of chromatic color space and consequently, skin color recognition is typically used to discover the face location instead of using other facial features (Patil and Patil, 2014). This progression is portray how non-skin area is rejected from a picture with the goal that the picture may contains just skin like zones which will be our skin color divided picture for further preparing (Raghuvanshi and Agrawal, 2012). The input image is RGB format which comprise 3 fundamental hues red, green and blue. The sensitivity of RGB color space for lighting makes it inefficient in splitting the skin color. For this reason, the image is converted to CIELAB color model to separate the chroma components (A and B). L component represents the luminance information which has more variation that is why discard this component (Kaur and Kranthi, 2012). This conversion is implemented by using "makecform" MATLAB function (Sujan et al., 2016).

Binary image: A binary image is a 2-dimensional matrix that contains just 0 's and 1 's values. The esteem 0 represents the pixels of black region and esteem 1 represents the pixels with white region. The skin color area takes the white color in the binary image. The MATLAB function "im $2 \mathrm{bw}$ " converts skin color segmentation image to binary image after apply the threshold on A and B components (Raghuvanshi and Agrawal, 2012).

Dismissal of non-face area: The resulting binary image contains a skin region. Human face features and region properties methods of a human face are used to decide which region is most probable to human face region (Raghuvanshi and Agrawal, 2012). Dismisses of non-face skin region from a binary image depends on height to width ratio. Height to width ratio of the skin region is compared with threshold value if it is less than the threshold value then this region will be rejected from the class of likely human faces. Region properties based on bounding box MATLAB function has been used to determine height to width ratio of every skin region (Patil and Patil, 2014).

\section{RESULTS AND DISCUSSION}

In this study, a comparison between 2 methods of face detection has been implemented. Two types of the most important face detection methods have been evaluated. The 1st method is the Viola-Jones meanwhile the second method is the CIELAB. The comparison has been implemented under MATLAB environment to evaluate the performance of these 2 methods. For fair comparison, both algorithms are subjected to 66 images. Since, many images are used for comparison, selected images are shown in Fig. 8.

Viola-Jones method succeeded to detect 58 images. Samples of the detected images based Viola-Jones method are shown in Fig. 9. Numbers of the images that are the Viola-Jones method failed to detect them are 8 images. Therefore, the success ratio based Viola-Jones method is $87.87 \%$, meanwhile, the failed ratio is $12.12 \%$.

Regarding to CIELAB method, Fig. 10 shows the same selected images. The number of the images that has been detected based CIELAB method is 31 images. Furthermore, 35 images have been not detected accurately based CIELAB method. Therefore, the success and the failed ratios are 46.96 and $53.03 \%$, respectively. The 

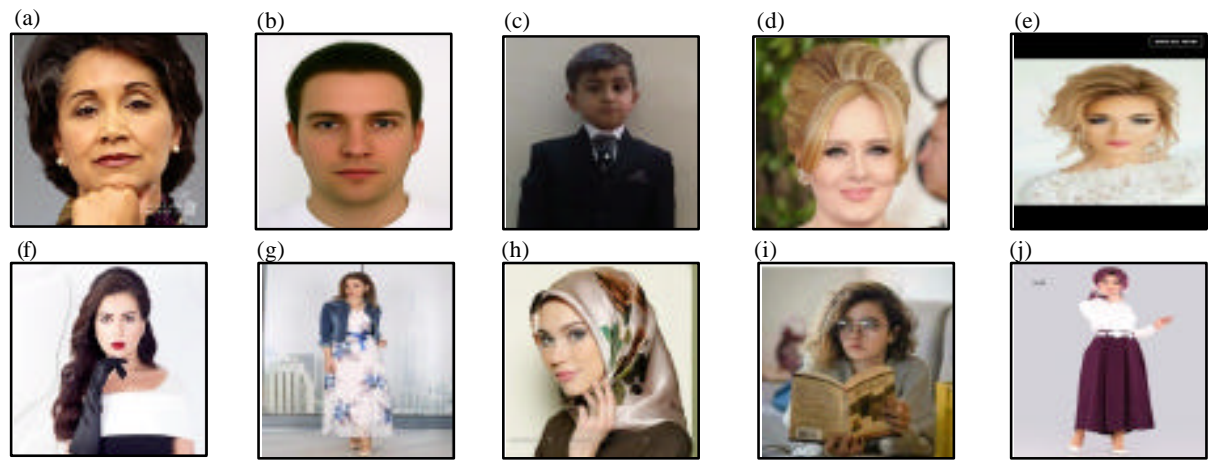

Fig. 8: a-j) Selected images from the comparison images
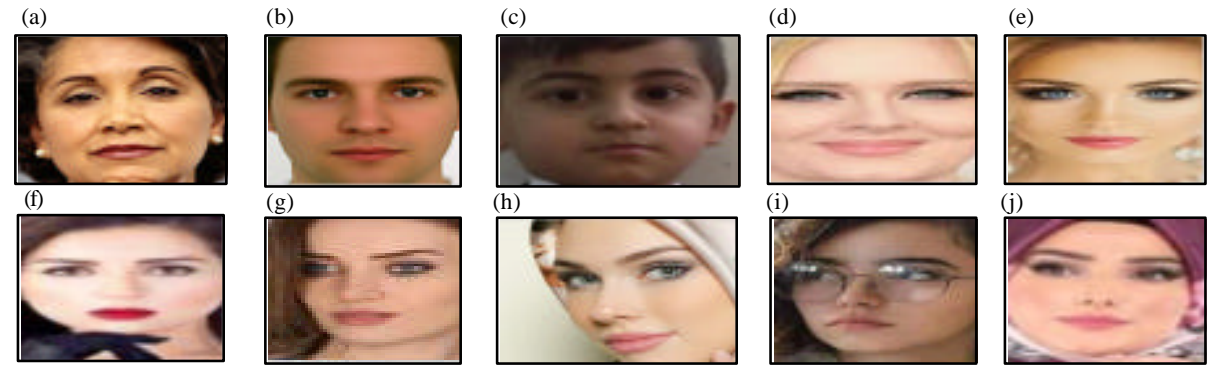

Fig. 9: a-j) Selected images based Viola-Jones method
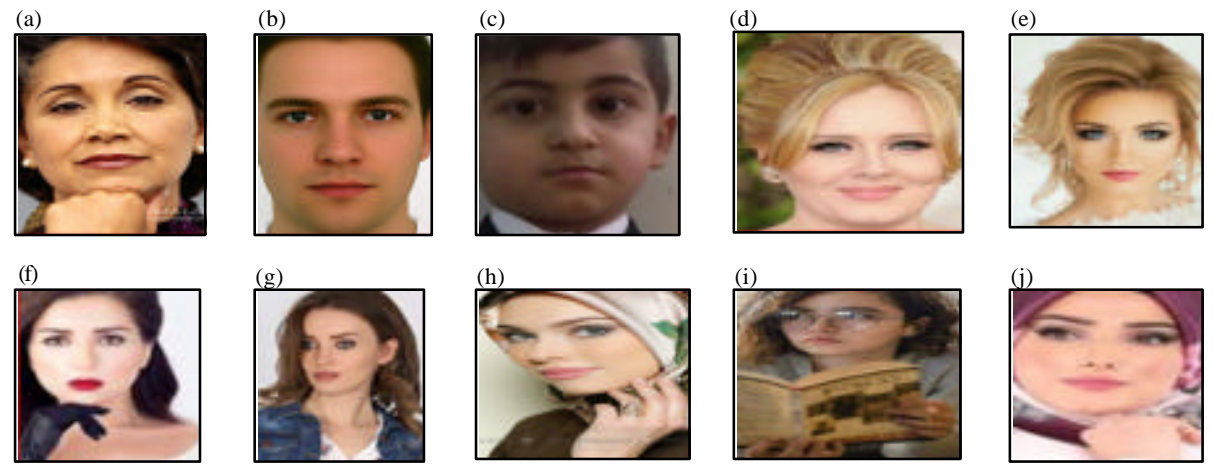

Fig. 10: a-j) Selected images based CIELAB method

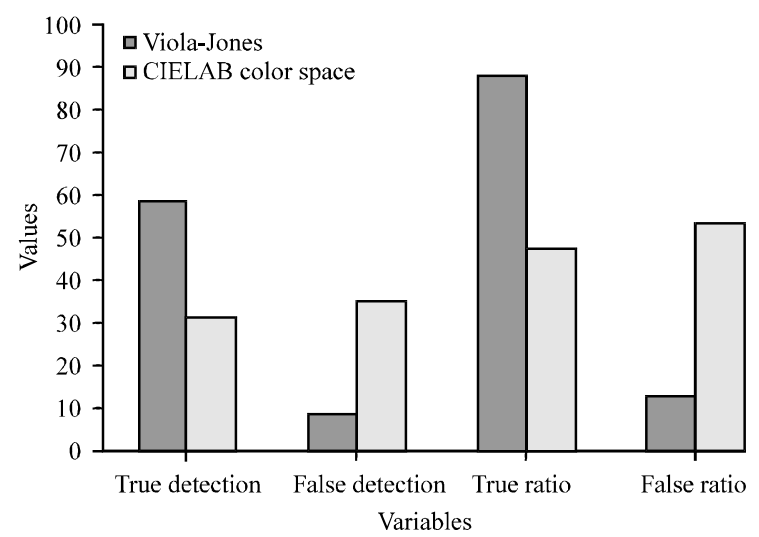

Fig. 11: Performance comparison of Viola-Jones and CIELAB
Table 1: Simulation Viola-Jones and CIELAB comparison

\begin{tabular}{lccccc}
\hline Methods & $\begin{array}{l}\text { Total } \\
\text { images }\end{array}$ & $\begin{array}{c}\text { Success } \\
\text { detection }\end{array}$ & $\begin{array}{c}\text { False } \\
\text { detection }\end{array}$ & $\begin{array}{c}\text { Success } \\
\text { ratio }(\%)\end{array}$ & $\begin{array}{c}\text { False } \\
\text { ratio }(\%)\end{array}$ \\
\hline Viola-Jones & 66 & 58 & 8 & 87.87 & 12.12 \\
CIELAB color space & 66 & 31 & 35 & 46.96 & 53.03 \\
\hline
\end{tabular}

comparison between the Viola-Jones and CIELAB is shown in Table 1. The results show clearly that the Viola-Jones method achieved high ratio face detection compare with the CIELAB method as can be shown in Fig. 11. Therefore, the using of the Viola-Jones method improves the system of the face detection.

\section{CONCLUSION}

Face detection becomes very important issue in many application systems. Therefore, performance evaluation of 
2 algorithms for face detection has been investigated in this study. These algorithms are Viola-Jones algorithm and CIELAB color space. The evaluation has been achieved under MATLAB environment. For fair comparison, 66 images have been subjected for both algorithms. The success detection of the face images based Viola-Jones and CIELAB are 58 and 31, respectively. Therefore, the success ratios are 87.87 and $46.96 \%$ for the Viola-Jones and CIELAB, respectively. The results clearly show that the Viola-Jones algorithm provide better performance than the other method. Furthermore, the Viola-Jones algorithm is more accurate to detect the face region; meanwhile the CIELAB algorithm may detect other skin region in image which is not belonging to the face. Moreover, Viola-Jones algorithm is more fast and robust. Therefore, the Viola-Jones algorithm is preferred for face detection.

\section{REFERENCES}

Anchit, A. and S. Mathur, 2014. Comparative analysis of haar and skin color method for face detection. Proceedings of the International Conference on Recent Advances and Innovations in Engineering (ICRAIE), May 9-11, 2014, IEEE, Jaipur, India, ISBN:978-1-4799-4040-0, pp: 1-5.

Dabhi, M.K. and B.K. Pancholi, 2016. Face detection system based on Viola-Jones algorithm. Intl. J. Sci. Res., 5: 62-64.

Dasan, M., A. Alqudah and O. Debeir, 2015. Face detection using Viola and Jones method and neural networks. Proceedings of the 2015 International Conference on Information and Communication Technology Research (ICTRC), May 17-19, 2015, IEEE, Abu Dhabi, UAE., ISBN:978-1-4799-8966-9, pp: 40-43.

Deshpande, N.T. and S. Ravishankar, 2017. Face detection and recognition using Viola-Jones algorithm and Fusion of PCA and ANN. Adv. Comp. Sci. Technol., 10: 1173-1189.

Fan, H. and W. Wang, 2013. Edge detection of color road image based on Lab model. Proceedings of the 20135 th International Conference on Computational and Information Sciences (ICCIS), June 21-23, 2013, IEEE, Shiyang, China, ISBN:978-0-7695-5004-6, pp: 298-301.

Gupta, A. and R. Tiwari, 2014. Face detection using modified Viola Jones algorithm. Intl. J. Recent Res. Math. Comput. Sci. Inf. Technol., 1: 59-66.

Gupta, V. and D. Sharma, 2014. A study of various face detection methods. Intl. J. Adv. Res. Comput. Commun. Eng., 3: 6694-6697.
Kaur, A. and B.V. Kranthi, 2012. Comparison between $\mathrm{YCbCr}$ color space and CIELAB color space for skin color segmentation. Intl. J. Appl. Inf. Syst., 3: 30-33.

Luh, G.C., 2014. Face detection using combination of skin color pixel detection and Viola-Jones face detector. Proceedings of the 2014 International Conference on Machine Learning and Cybernetics (ICMLC) Vol. 1, July 13-16, 2014, IEEE, Lanzhou, China, ISBN:978-1-4799-4216-9, pp: 364-370.

Lv, C., Zhang, T. and C. Lin, 2017. Face detection based on skin color and AdaBoost algorithm. Proceedings of the 2017 29th International Conference on Chinese Control And Decision (CCDC), May 28-30, 2017, IEEE, Chongqing, China, ISBN:978-1-5090-4658-4, pp: 1363-1367.

Manjare, M.S. and M.S. Chougule, 2013. Skin detection for face recognition based on HSV color space. Intl. J. Eng. Sci. Res. Technol., 2: 1883-1887.

Mehta, P. and P. Tomar, 2016. An efficient attendance management sytem based on face recognition using Matlab and raspberry Pi 2. Intl. J. Eng. Technol. Sci. Res., 3: 71-78.

Nehru, M. and S. Padmavathi, 2017. Illumination invariant face detection using viola jones algorithm. Proceedings of the 2017 4th International Conference on Advanced Computing and Communication Systems (ICACCS), January 6-7, 2017, IEEE, Coimbatore, India, ISBN:978-1-5090-4560-0, pp: 1-4.

Patil, G.A. and S.A. Patil, 2014. Face detection using RGB ratio model. Intl. J. Comput. Appl., 2014: 18-20.

Prashanth, K.G. and M. Shashidhara, 2014. Real time detection and tracking of human face using skin color segmentation and region properties. Intl. J. Image Graphics Sign. Proc., 6: 40-46.

Raghuvanshi, D.S. and D. Agrawal, 2012. Human face detection by using skin color segmentation, face features and regions properties. Intl. J. Comput. Appl., 38: 14-17.

Raya, I.G.N.M.K., A.N. Jati and R.E. Saputra, 2017. Analysis realization of Viola-Jones method for face detection on CCTV camera based on embedded system. Proceedings of the 2017 International Conference on Robotics, Biomimetics and Intelligent Computational Systems (Robionetics), August 23-25 2017, IEEE, Bali, Indonesia, ISBN:978-1-5386-2885-0, pp: 1-5.

Shaik, K.B., P. Ganesan, V. Kalist, B.S. Sathish and J.M.M. Jenitha, 2015. Comparative study of skin color detection and segmentation in HSV and $\mathrm{YCbCr}$ color space. Proc. Comput. Sci., 57: 41-48. 
Sujan, M., N. Alam, S.A. Noman and M.J. Islam, 2016. A segmentation based automated system for brain tumor detection. Intl. J. Comput. Appl., 153: 41-49.

Tikoo, S. and N. Malik, 2017. Detection of face using Viola Jones and recognition using back propagation neural network. Comput. Vision Pattern Recognit., 5: 288-295.
Viola, P. and M. Jones, 2001. Rapid object detection using a boosted cascade of simple features. Proceedings of the 2001 IEEE Computer Society Conference on Computer Vision and Pattern Recognition(CVPR 2001) Vol. 1, December 8-14, 2001, IEEE, Kauai, Hawaii, USA., ISBN:0-7695-1272-0, pp: I-511-I-518. 